\title{
Radiation Response of Nanostructured Pyrochlore and Zirconia
}

\author{
Jie Lian, * Jiaming Zhang, ** Antonio F. Fuentes, *** Fereydoon Namavar, **** R. C. Ewing** \\ * Department of Mechanical, Aerospace \& Nuclear Engineering, Rensselaer Polytechnic Institute, \\ Troy, NY 12180 \\ ** Departments of Geological Sciences and Materials Science \& Engineering, University of \\ Michigan, Ann Arbor, Michigan 48109-1005, USA \\ *** Instituto de Ciencia de Materiales de Aragon, Universidad de Zaragoza, 50009 Zaragoza, Spain \\ **** University of Nebraska Medical Center, Omaha, NE 68198, USA
}

Fluorite-related structures, including pyrochlore and zirconia, have a wide range of important technological applications varying from electrolyte for solid oxide fuel cells to potential matrix used as advanced fuel and waste forms for nuclear applications. Nanocrystalline zirconia displays superior mechanical, electrical, and thermal properties as compared with its conventional coarsened bulk counterpart. It is of both scientific interest and technical importance to understand how nanostructured pyrochlore and zirconia behave under intense radiation environment. We have synthesized a wide range of pyrochlore and zirconia nanostructured materials with well controlled grain size and polymorphs by high energy ball milling and ion beam assisted deposition (IBAD) approaches, respectively, and investigated the phase stability, microstructure evolution and structural transformation occurred in these nanostructured materials upon energetic beam bombardment.

Significantly enhanced resistance to amorphization has been observed for nanostructured pyrochlore oxides with the composition of $\mathrm{Gd}_{2}\left(\mathrm{Ti}_{0.65} \mathrm{Zr}_{0.35}\right)_{2} \mathrm{O}_{7}$ as the crystal size is less than $20 \mathrm{~nm}$ (Fig. 1), and this result highlights an important example that nanostructured materials enhance radiation performance. By controlling the thermal annealing time and temperature, the crystal size and the degree of structural disordering can be well controlled, which allow us to investigate how grain size and the degree of structural disordering affect materials performance independently. Radiation resistance is not inherent for nanostructured materials, and similar to that observed in bulk pyrochlore, the radiation tolerance of nanostructured pyrochlores are highly compositionaldependent. For example, pure $\mathrm{Gd}_{2} \mathrm{Ti}_{2} \mathrm{O}_{7}$ nanostructures can be easily amorphized by ion beam irradiations despite that crystal size is smaller than $20 \mathrm{~nm}$. In addition to the size effect, the degree of structural disordering significantly affects the phase stability of nanostructured materials under irradiation conditions. $\mathrm{Y}_{2} \mathrm{Ti}_{2} \mathrm{O}_{7}$ nanostructures with a greater degree of ordering display a greater tolerance to ion beam-induced amorphization. Behaviors of nano-scale pyrochlore oxides under irradiation are intriguing, and the interplay of among composition, size, bond nature, degree of disordering defines the structural deviation and defect energetics that may essentially control phase stability of pyrochlore materials under irradiation conditions.

The phase stability of nanocrystalline zirconia polymorphs (amorphous, tetragonal, cubic and monoclinic) was also investigated by energetic beam bombardment. An ion beam-induced amorphous-to-tetragonal phase transformation occurred upon $1 \mathrm{MeV} \mathrm{Kr}^{2+}$ at room temperature, and the crystal size can be well controlled from $5 \mathrm{~nm}$ up to $40 \mathrm{~nm}$. Furthermore, we also found that monoclinic nano-zirconia transforms to a tetragonal phase upon ion irradiation, suggesting that tetragonal phase is more energetically favorable than monoclinic or amorphous phase at the nanosize regime under irradiation conditions. A greatly enhanced resistance toward grain coarsening has been observed for nanostructured cubic zirconia upon ion beam bombardment. A previous study reported that nanostructured tetragonal zirconia nanocrystals can be easily damaged by ion beam irradiation as the size is below $3 \sim 5 \mathrm{~nm}$, in which the excess surface energy associated with the nano- 
scaled zirconia may dominate the phase transformation process. The reversible phase transformation between amorphous and tetragonal polymorphs for nanostructured zirconia has a significant impact for controlling the phase stability and tailoring materials physical properties in this important nanosystem. The phase stability of different zirconia polymorphs under intense irradiation environment essentially depends on the grain size, surface and interface geometry and configuration.

These results demonstrate a great potential of using ion beam techniques to tailor materials properties by controlling phase stability and manipulating the microstructure at the nano-scale, and form the scientific basis for developing advanced materials with enhanced performance through the integration of nano-scale design strategy.
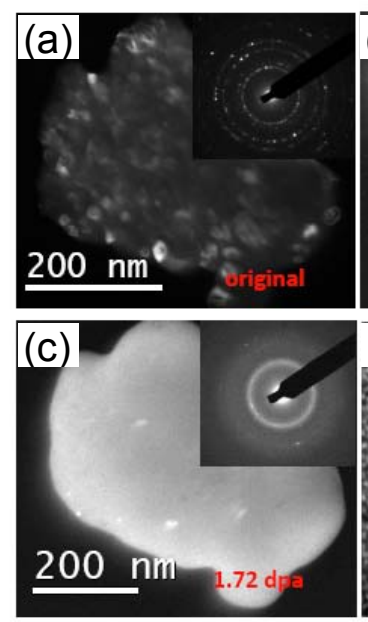
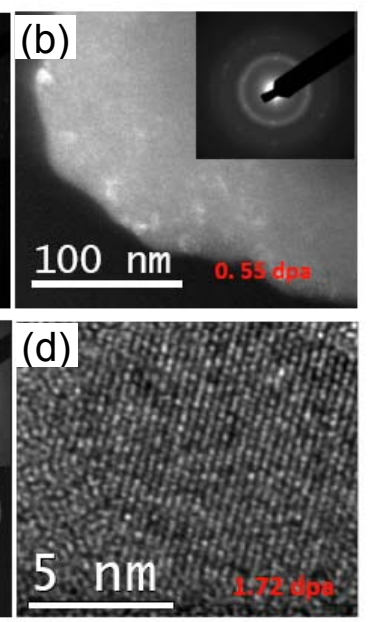

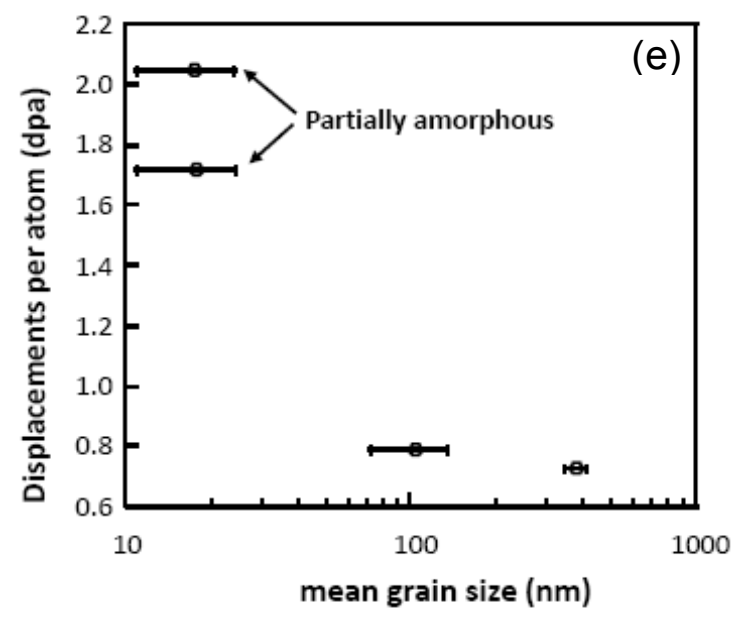

FIG. 1. Microstructural evolution of nanostructured $\left.\mathrm{Gd}_{2} \mathrm{Ti}_{0.65} \mathrm{Zr}_{0.35}\right)_{2} \mathrm{O}_{7}(\mathrm{HEBM}$ and annealing at $1000{ }^{\circ} \mathrm{C}$ for $12 \mathrm{hrs}$ ) upon $1 \mathrm{MeV} \mathrm{Kr}^{2+}$ irradiation at room temperature (a d) and enhanced amorphization resistance as a function of decreasing grain size (e) (Lian et al., in preparation).
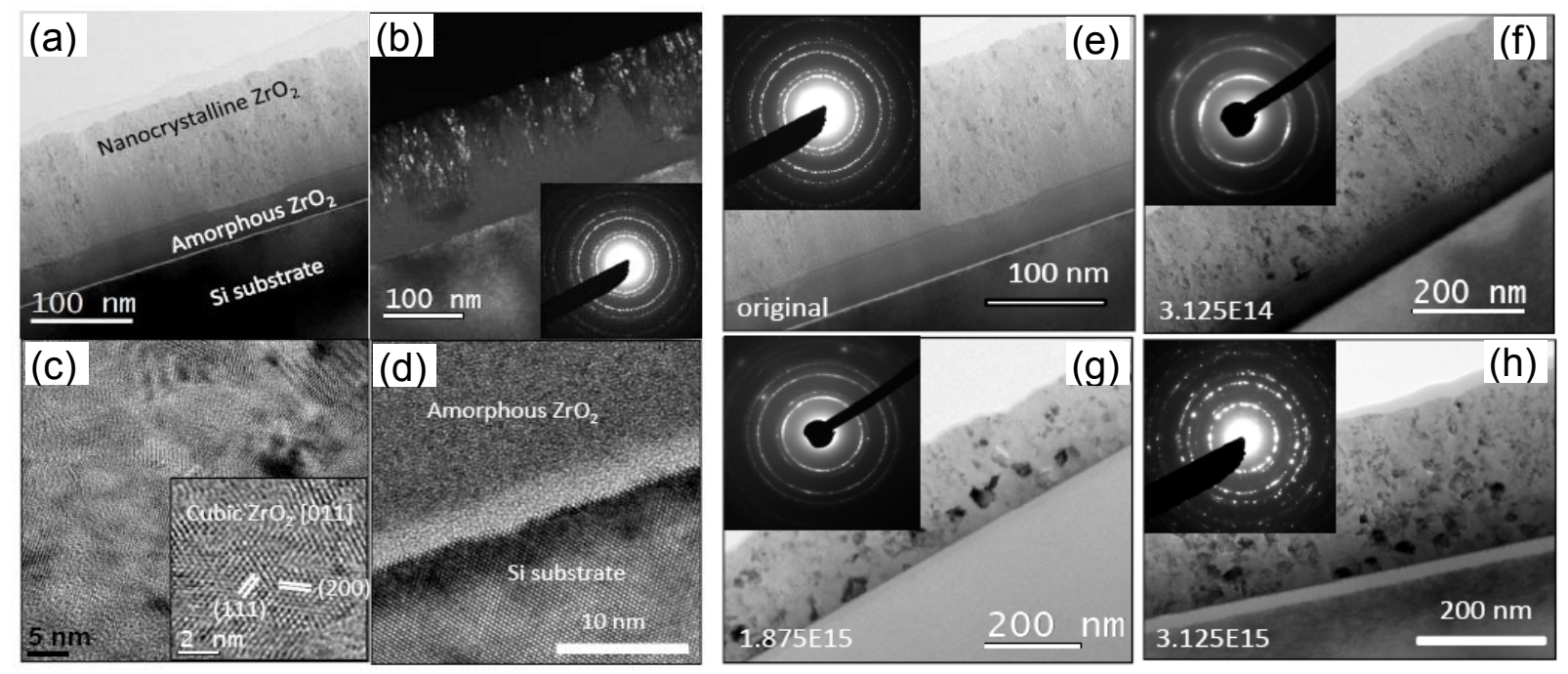

FIG. 2. Cross-sectional TEM images of a bilayer (amorphous and cubic) zirconia nano-film fabricated by IBAD method (a d) and an ion beam-induced amorphous-to-tetragonal phase transformation and grain coarsening in nanostructured zirconia upon $1 \mathrm{MeV} \mathrm{Kr}^{2+}$ irradiation at room temperature as a function of ion fluence (e-h) (Lian et al., submitted). 